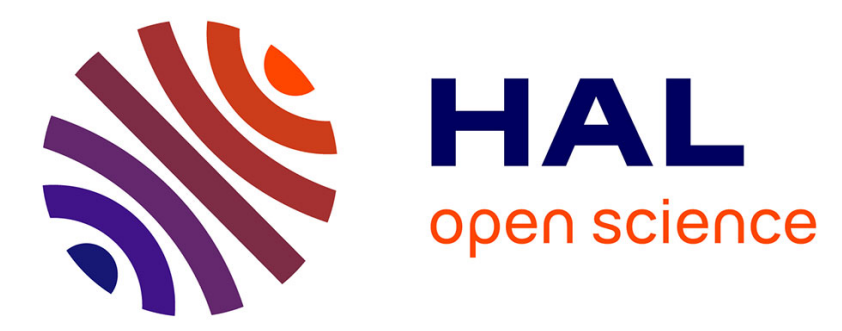

\title{
Efficient networks for a class of games with global spillovers
}

Pascal Billand, Christophe Bravard, Jacques Durieu, Sudipta Sarangi

\section{To cite this version:}

Pascal Billand, Christophe Bravard, Jacques Durieu, Sudipta Sarangi. Efficient networks for a class of games with global spillovers. 2015. halshs-01247683

\section{HAL Id: halshs-01247683 \\ https://shs.hal.science/halshs-01247683}

Preprint submitted on 22 Dec 2015

HAL is a multi-disciplinary open access archive for the deposit and dissemination of scientific research documents, whether they are published or not. The documents may come from teaching and research institutions in France or abroad, or from public or private research centers.
L'archive ouverte pluridisciplinaire $\mathbf{H A L}$, est destinée au dépôt et à la diffusion de documents scientifiques de niveau recherche, publiés ou non, émanant des établissements d'enseignement et de recherche français ou étrangers, des laboratoires publics ou privés. 
UMR 5824

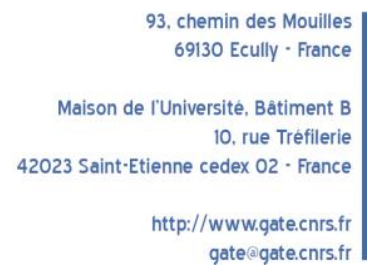

WP 1537 - December 2015

\title{
Efficient networks for a class of games with global spillovers
}

Pascal Billand, Christophe Bravard, Jacques Durieu, Sudipta Sarangi

\begin{abstract}
:
In this paper we examine efficient networks in network formation games with global spillovers that satisfy convexity and sub-modularity properties. Unlike the previous literature we impose these properties on individual payoff functions. We establish that efficient networks of this class of games are nested split graphs. This allows us to complete the work of Goyal and Joshi (2006) and Westbrock (2010) on collaborative oligopoly networks.
\end{abstract}

\section{Keywords:}

networks, efficiency, convexity, sub-modularity, oligopolies.

JEL codes:

C70, D85

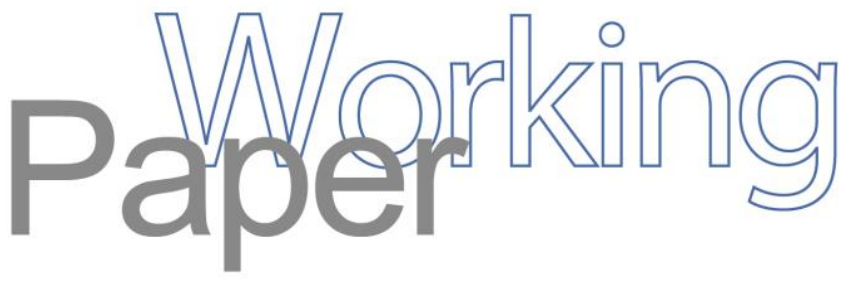




\title{
Efficient networks for a class of games with global spillovers*
}

\author{
PASCAL BILLAND ${ }^{a}$, CHRISTOPHE BRAVARD $^{b}$, JACQUES DURIEU $^{b}$, SUDIPTA SARANGI $^{c}$
}

${ }^{a}$ Université de Lyon, Lyon, F-69007, France ; CNRS, GATE Lyon Saint-Etienne, Ecully, F-69130, France ; Université Jean Monnet, Saint-Etienne, F-42000, France. Email: pascal.billand@univ-stetienne.fr,

${ }^{b}$ Univ. Grenoble Alpes and INRA, UMR 1215 GAEL, F-38000 Grenoble, France; CREG ; GATE Lyon St Etienne. Email: christophe.bravard@univ-st-etienne.fr, jacques.durieu@univ-st-etienne.fr, ${ }^{c}$ DIW Berlin and Department of Economics, Virginia Tech, Blacksburg, VA 24061 USA. email: ssarangi@vt.edu

\begin{abstract}
In this paper we examine efficient networks in network formation games with global spillovers that satisfy convexity and sub-modularity properties. Unlike the previous literature we impose these properties on individual payoff functions. We establish that efficient networks of this class of games are nested split graphs. This allows us to complete the work of Goyal and Joshi (2006) and Westbrock (2010) on collaborative oligopoly networks.
\end{abstract}

JEL Classification: C70, D85.

Key Words: networks, efficiency, convexity, sub-modularity, oligopolies.

\section{Introduction}

Connections between agents play a crucial role both at the individual level as well as in the aggregate. For example, it has been argued that better connected agents have higher

${ }^{*}$ We thank the Associate Editor Takahashi Satoru and two anonymous referees for their useful comments. Any opinion, findings, and conclusions or recommendations expressed in this material are those of the authors and do not necessarily reflect the views of the National Science Foundation. 
probabilities of finding a job (Granovetter, 1974, Calvó-Armengol, Jackson, 2004). Similarly, firms use their collaborations to obtain a degree of competitive advantage on the market (Hagedoorn, 2002; Powell et al., 2005). Recent empirical findings establish that in many social and economic networks, the distribution of connections is very unequal across individuals. Goyal and Joshi (2006; henceforth GJ) design a strategic model of network formation to study these issues. They propose a framework that takes into account externalities created by links. The authors study the relationship between different types of externalities and the inequality in links in equilibrium networks. In particular, GJ use a payoff function for each player $i$ which depends on the number of links formed by player $i$ and the total number of links formed by other players. They examine the architecture of equilibrium networks based on the variation of the marginal payoffs arising from the changing of the number of links formed by player $i$ and the changing of the total number of links formed by other players.

One of the most interesting case they deal with concerns the situation where the marginal payoff function of player $i$ is increasing in the number of links formed by player $i$ (the payoff function is convex in the number of links formed by player $i$ ) and decreasing in the total number of links formed by other players (the payoff function is sub-modular ${ }^{1}$ ). It is worth noting that the model of Cournot oligopoly with cost reduction analyzed by GJ (2003) satisfies the properties of this general framework. The authors propose a game of network formation in a homogeneous-product oligopoly. In the first stage of the game, firms form bilateral collaborative links. These links allow firms to decrease their marginal costs. In the second stage, all firms compete in the product market. GJ (2003) examine stable networks, that is networks where no pair of unlinked firms has an incentive to form a link, and no firm has an incentive to remove any of its links unilaterally. They find that equilibrium networks contain a dominant group (a subset of firms connected together) while other firms are isolated.

Although stable networks are important, network efficiency is a major performance criterion for network designers or planers, and plays a prominent role in the traditional network literature. Consequently, studying efficient networks in a general class of network formation games that satisfy the convexity and sub-modularity properties is an important task. In this paper, we examine efficient networks when the payoff function of each player $i$ is convex in the

\footnotetext{
${ }^{1}$ Sub-modularity property is called strategic substitute property in GJ (2006).
} 
number of links formed by $i$ and sub-modular or super-modular. A caveat is needed here - to obtain results about efficiency, we require an additional convexity condition on the payoffs of the players: the payoffs of each player are convex in the number of links in which she is not involved. This assumption allows us to take into account the impact of an additional link on the payoffs of players who are not involved in it.

Two papers have dealt with efficient networks in frameworks which satisfy the properties of convexity and sub-modularity. Westbrock (2010) examines efficient networks, that is networks which maximize social welfare, in a framework based on the two-stage GJ's (2003) model. He shows that non-empty and non-complete efficient networks are interlinked stars (Proposition 1, pg. 358, 2010), ${ }^{2}$ that is networks where all firms that are involved in the largest number of links are connected with all non-isolated firms. Moreover, he establishes conditions under which stable and efficient networks coincide. Billand et al. (2014) propose a model where firms form collaborative links in a multi-market framework. They establish that efficient networks belong to a class of networks called nested split graphs (NSG). ${ }^{3}$ In NSG, neighborhood of players $^{4}$ are nested. More precisely, if a player $i$ has formed more links than player $j$, then all the neighbors of $j$ are in the neighborhood of $i$.

In this paper, we focus on efficient networks, where the total payoffs are maximized and global efficient networks, where the total payoff function of the players plus another function is maximized. This additional function could be used to capture payoffs of those who are not players in the game itself. In the context of oligopolies for example, this additional function could be used to capture consumer surplus. Our first result establishes that in an efficient network $G$, if players $i$ and $i^{\prime}$ are linked, then any two players $j$ and $j^{\prime}$, such that $j$ has at least as many links as $i$, and $j^{\prime}$ has at least as many links as $i^{\prime}$, are linked in $G$ (Proposition 1 ) and provide a procedure for checking whether a particular graph is a candidate for being an efficient network. As a corollary we show that both efficient and global efficient networks are interlinked stars. Second, we observe that networks which satisfy this property are NSG (Proposition

\footnotetext{
${ }^{2}$ Westbrock shows that an efficient network has either a dominant group architecture, or an interlinked star architecture (Proposition 1, pg. 358, 2010). In this paper, we modify the definition of interlinked stars slightly: with our definition networks that have a dominant group architecture have also an interlinked star architecture.

${ }^{3}$ To the best of our knowledge three other papers have used NSG in the network formation literature, König et al. (2012), König et al. (2011), and Belhaj et al. (2013).

${ }^{4}$ The neighbors of player $i$ are the players with whom player $i$ is linked.
} 
2). Third, we establish that if the sub-modularity property is sufficiently low relative to the convexity properties, then efficient networks are either empty or complete (Proposition 3). In that case, players are in symmetric position in efficient networks. Finally, we show that if each player's payoff function satisfies convexity properties and super-modularity, then efficient networks are either empty or complete (Proposition 4). We propose several examples in this paper that allow us to describe models where our results can be applied: models of oligopoly (with homogeneous or differentiated goods) where links lead to cost reduction and a model of public good provision.

Our paper adds to the literature in several ways. First, it complements the stable network results of GJ (2006) by identifying efficient networks with the convexity and sub-modularity or super-modularity properties. Second, we extend Westbrock (2010). We refine his results concerning the architecture of efficient networks by relating NSG to the architecture found by Westbrock (interlinked stars). ${ }^{5}$ Moreover, we provide a simple method allowing us to check whether a network is a candidate for being an efficient network. Another crucial difference is that we do not use the same approach as Westbrock. While Westbrock provides general conditions on the total welfare function ${ }^{6}$ to obtain his results, we provide general conditions on the individual payoff functions. This makes our results interesting for comparison purposes since stable networks are typically identified using conditions on individual payoffs. Third, we extend Billand et al. (2014) by identifying the properties that allow us to obtain NSG as efficient networks in their framework. Indeed their model is computationally intensive, making it difficult to understand what drives their results. In this paper, we specifically examine the role of each of the convexity properties and sub-modularity in leading to different efficient networks.

The paper is organized as follows. In section 2, we introduce the model and the basic properties of the payoff function. We then show that the basic model of GJ (2003) satisfies these properties. We also define the architectures used in this paper. In section 3, we establish our main result: efficient and global efficient networks are NSG. We also examine the role played by sub-modularity. In section 4 , we discuss the role played by the costs of forming links on the efficient networks for a specific payoff function.

\footnotetext{
${ }^{5}$ Interlinked stars are not always NSG.

${ }^{6}$ Westbrock (2010) defines a specific convexity property on the aggregate social welfare function.
} 


\section{Model setup}

\section{$2.1 \quad$ Networks}

To simplify notation, we use $\llbracket a, b \rrbracket=\{a, a+1, \ldots, b\}$ for $a, b \in \mathbb{N}$ and denote by $\lfloor x\rfloor$ the largest integer not greater than $x$. Let $N=\llbracket 1, n \rrbracket$ be the set of players, with $i$ and $j$ as typical members of $N$. For any $i, j \in N$, the pair-wise relationship between the two players is captured by a binary variable, $G_{i, j} \in\{0,1\} ; G_{i, j}=1$ means that a link, $i j$, exists between players $i$ and $j, G_{i, j}=0$ means that there is no link between $i$ and $j$. A network $G=\left\{\left(G_{i, j}\right)_{i \in N, j \in N}\right\}$ is a formal description of the links that exist between the players. The number of links in $G$ is denoted by $g$. Network $G_{-i}$ is similar to network $G$, except that player $i$ and all her links are removed; the number of links in $G_{-i}$ is denoted by $g_{-i}$. The set of players with whom player $i$ is linked in $G$ is denoted by $G_{i}$ and its cardinality, $g_{i}$, is called the degree of $i$. We denote by $G^{-X}$ the network obtained when we delete all the players in $X \subset N$ and their links from the network $G .{ }^{7}$ Let $\mathcal{G}$ denote the set of all simple networks when there are $n$ players, that is networks without loops (player $i$ cannot form a link with herself) or multiple links (players $i$ and $j$ can establish at most one link between them). Let $G+i j$ denote the network obtained by replacing $G_{i, j}=0$ in network $G$ by $G_{i, j}=1$. Similarly, let $G-i j$ denote the network obtained by replacing $G_{i, j}=1$ in network $G$ by $G_{i, j}=0$.

We now define the main network configurations that are used extensively in our model. An empty network is a network where all players are isolated. In order to define interlinked stars and nested split graphs (NSG), we introduce a partition of the players of any network as follows. Let $G$ be a network whose distinct positive player-degrees are $\delta_{1}<\ldots<\delta_{m}$, and let $\delta_{0}=0$ (even if no player of degree 0 exists). Let $D_{\ell}=\left\{i \in N: g_{i}=\delta_{\ell}\right\}$ for $\ell \in \llbracket 0, m \rrbracket$. The sequence $D_{0}, \ldots, D_{m}$ is called the degree partition of $G$. Westbrock (2010) defines a class of networks which plays a crucial role in our analysis: the class of interlinked stars. We modify the definition of this class of networks in order to simplify the presentation. ${ }^{8}$ In an interlinked star $G$, each player $i \in D_{m}$ is linked with each player $j$ such that $g_{j}>0$. We now define the

\footnotetext{
${ }^{7}$ Observe that if $X$ is the singleton $\{i\}$, then $G^{\{-i\}}=G_{-i}$, we use the latter for this case.

${ }^{8}$ In the original definition given by Westbrock (2010), an interlinked star $G$ contains at least two groups of players with strictly positive degrees. A network where there is only one group of players who have formed links together while other players are isolated is called a network with the dominant group architecture (see network $G^{\prime}$ in Figure 3 ) by Westbrock. To sum up with our definition interlinked stars are both the interlinked stars of Westbrock and the networks which have the dominant group architecture.
} 
key architecture of this paper: the NSG.

Definition 1 (Mahadev and Peled, Theorem 1.2.4, pg. 10, 1995). Consider a nested split graph $G$. For each player $i \in D_{\ell}, \ell \in \llbracket 1, m \rrbracket$,

$$
G_{i}= \begin{cases}\bigcup_{j=1}^{\ell} D_{m+1-j}, & \text { if } \ell=1, \ldots,\left\lfloor\frac{m}{2}\right\rfloor, \\ \bigcup_{j=1}^{\ell} D_{m+1-j} \backslash\{i\}, & \text { if } \ell=\left\lfloor\frac{m}{2}\right\rfloor+1, \ldots, m .\end{cases}
$$

In Figure 1, we illustrate a NSG: A line between $D_{\ell}$ and $D_{\ell^{\prime}}$ indicates that every player of $D_{\ell}$ is linked with every player of $D_{\ell^{\prime}}$. The rectangle indicates that the included players are linked together.

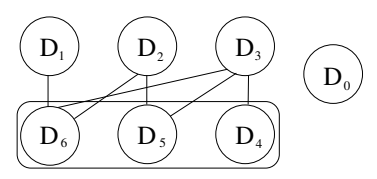

Figure 1: A NSG

\subsection{Payoffs}

We consider network formation games with global spillovers, that is games in which the marginal returns from links for every player can be expressed in terms of the number of links of the player and the sum of links of the rest of the players. This class of games has been examined by GJ (2006). Let $\phi: \llbracket 0, n-1 \rrbracket \times \llbracket 0,(n-2)(n-1) / 2 \rrbracket \rightarrow \mathbb{R}$ be a function. We set $\phi_{1}(x, y)=\phi(x, y)-\phi(x-1, y)$ and $\phi_{2}(x, y)=\phi(x, y)-\phi(x, y-1)$. We define the payoff function of each player $i$ as follows:

$$
\pi_{i}(G)=\phi\left(g_{i}, g_{-i}\right)
$$

We assume that $\phi$ satisfies the following properties.

Property 1. $\phi$ is convex in its first argument if for all $x \in \llbracket 1, n-2 \rrbracket$ and for all $y \in$ $\llbracket 0,(n-2)(n-1) / 2 \rrbracket, \phi_{1}(x+1, y) \geq \phi_{1}(x, y)$. 
Property 2. $\phi$ is strictly convex in its second argument if for all $x \in \llbracket 0, n-1 \rrbracket$ and for all $y \in \llbracket 1,[(n-2)(n-1) / 2]-1 \rrbracket, \phi_{2}(x, y+1)>\phi_{2}(x, y)$.

Property 3. $\phi$ is sub-modular if for all $x \in \llbracket 0, n-2 \rrbracket$, for all $y, y^{\prime} \in \llbracket 0,[(n-2)(n-1) / 2] \rrbracket$ and $y \geq y^{\prime}, \phi_{1}(x+1, y) \leq \phi_{1}\left(x+1, y^{\prime}\right) \cdot{ }^{9}$

Our results mainly concern situations where $\phi$ is sub-modular. However, sometimes we assume that $\phi$ is super-modular. We say that $\phi$ is super-modular if for all $x \in \llbracket 0, n-2 \rrbracket$, for all $y, y^{\prime} \in \llbracket 0,[(n-2)(n-1) / 2]-1 \rrbracket$ and $y \geq y^{\prime}, \phi_{1}(x+1, y) \geq \phi_{1}\left(x+1, y^{\prime}\right)$.

In the rest of the paper we are particularly interested in specific payoff functions. Let $\theta:(x, y) \mapsto(a x+b y+c)^{2}-f(x)$ be a function where $a, b, c \in \mathbb{R}$ and $f$ is a concave function. It is worth noting that if the quadratic form $(a x+b y)^{2}$ satisfies Properties 1,2 and 3 , then function $\theta$ satisfies Properties 1, 2 and 3. Moreover, the quadratic form $(a x+b y)^{2}$ satisfies Properties 1,2 and 3 if $\operatorname{sign}(a) \neq \operatorname{sign}(b)$, and $b>0 .{ }^{10}$ We now present an economic example to illustrate the choice of this payoff formulation and Properties 1, 2 and 3.

Example 1.a Cournot Oligopoly with Cost Reduction (GJ, 2003). This example is taken from GJ (2003). Consider a homogeneous product Cournot oligopoly consisting of $n$ firms which face the linear inverse demand function: $p=\alpha-\sum_{i \in N} q_{i}$, where $p$ is the price of the product, $q_{i}$ is the quantity produced by firm $i$, and $\alpha>0$. The firms have zero fixed costs and constant returns-to-scale cost functions. When firms enter into bilateral collaborations, they lower their marginal costs. More precisely, the marginal cost of firm $i$ is given by $C\left(g_{i}\right)=\gamma_{0}-\gamma g_{i}$, with $\gamma_{0}>(n-1) \gamma>0$. Given any network $G$, the Cournot equilibrium output of firm $i \in N$ can be written as:

$$
q_{i}(G)=\frac{\alpha-\gamma_{0}+(n-1) \gamma g_{i}-2 \gamma g_{-i}}{n+1}
$$

\footnotetext{
${ }^{9}$ Recall that this property is called strategic substitute property in GJ (2006).

${ }^{10}$ We note that the three properties are preserved if $f$ is convex and $f(x)=a^{\prime} x^{2}+b^{\prime} x+c^{\prime}$, with $0<a^{\prime}<a$, $b^{\prime}, c^{\prime} \in \mathbb{R}$.
} 
with $\alpha-\gamma_{0}-(n-1)(n-2) \gamma>0$ to ensure that each firm produces a strictly positive quantity in equilibrium. Let $f: g_{i} \mapsto f\left(g_{i}\right)$ be a function which measures the costs of forming links; we assume that $f$ is concave, that is for all $g_{i} \in \llbracket 2, n-2 \rrbracket$, we have $f\left(g_{i}+1\right)-f\left(g_{i}\right) \leq f\left(g_{i}\right)-f\left(g_{i}-1\right)$. The Cournot profits for firm $i \in N$ are given by $\pi_{i}(G)=\left(q_{i}(G)\right)^{2}-f\left(g_{i}\right)$. This means that $\pi_{i}(G)=\phi\left(g_{i}, g_{-i}\right)$. Since $\operatorname{sign}(\gamma(n-1 / n+1)) \neq \operatorname{sign}(-2 \gamma / n+1), \phi$ satisfies Properties 1,2 and 3.

It is worth noting that in the above example we assume that the costs of forming links are a concave function (it can also be a convex function as defined in footnote 10). Hence, we generalize the basic model of GJ (2003) where the cost of forming links is an affine function. Note also that Properties 1, 2, and 3 are preserved in a linear differentiated product oligopoly consisting of $n$ firms that compete either in quantities, or in prices.

Example 2.a Differentiated Oligopolies with Cost Reduction (Westbrock, 2010). Suppose that each firm $i$ faces the following linear inverse demand function: $p_{i}=\alpha-q_{i}-\beta \sum_{j \in N \backslash\{i\}} q_{j}$, where $p_{i}$ is the price of the product sold by firm $i, \alpha>0$, and $\left.\beta \in\right] 0,1[$.

The Cournot equilibrium output of firm $i \in N$ can be written as

$$
q_{i}^{C}(G)=\frac{(2-\beta)\left(\alpha-\gamma_{0}\right)+\gamma((n-3) \beta+2) g_{i}-2 \gamma \beta g_{-i}}{(2-\beta)(2+\beta(n-1))} .
$$

The Cournot profits for firm $i \in N$ are given by $\left.\pi_{i}^{C}(G)=\phi\left(g_{i}, g_{-i}\right)\right)=\left(q_{i}^{C}(G)\right)^{2}-f\left(g_{i}\right)$. Since $\operatorname{sign}(\gamma((n-3) \beta+2))((2-\beta)(2+\beta(n-1))) \neq \operatorname{sign}(2 \gamma \beta)((2-\beta)(2+\beta(n-1))), \pi_{i}^{C}(G)$ satisfies Properties 1, 2, and 3.

In the Bertrand equilibrium, the profits for firm $i \in N$ can be written in the form: $\pi_{i}^{B}(G)=$ $\phi\left(g_{i}, g_{-i}\right)=\left(a g_{i}+b g_{-i}+c\right)^{2}-f\left(g_{i}\right)$ where

$$
\begin{aligned}
& a=\lambda^{\frac{1}{2}} \gamma \frac{2+(5 n-11) \beta+\left(4 n^{2}-19 n+21\right) \beta^{2}+\left(\left(n^{2}-8 n+19\right) n-14\right) \beta^{3}}{(2+(n-3) \beta)(1+(n-1) \beta)(1-\beta)(2+(2 n-3) \beta)} \\
& b=-\lambda^{\frac{1}{2}} 2 \gamma \frac{\beta+(2 n-4) \beta^{2}+\left(n^{2}-4 n+4\right) \beta^{3}}{(2+(n-3) \beta)(1+(n-1) \beta)(1-\beta)(2+(2 n-3) \beta)} .
\end{aligned}
$$


and

$$
\lambda=\frac{(1-\beta)(1+(n-1) \beta)}{1+(n-2) \beta} .
$$

Since $\operatorname{sign}(a) \neq \operatorname{sign}(b), \pi_{i}^{B}(G)$ satisfies Properties 1, 2, and 3 .

Similarly, the model of multi-market oligopolies with product quality improvement (Billand et al., 2014) satisfies Properties 1, 2 and 3 when firms are able to form links only on one market.

\subsection{Efficient Networks}

Let $W: G \mapsto W(G)=\sum_{i \in N} \phi\left(g_{i}, g_{-i}\right)$ be a function. We call $W$ the total payoff function. Moreover, let $U: G \mapsto U(G)$ be another function. We say that $U$ is architecture independent if it satisfies the following property: If $G$ and $G^{\prime}$ satisfy $g=g^{\prime}$, then $U(G)=U\left(G^{\prime}\right)$.

Consider a function $\psi: g \mapsto \psi(g)$, and let $U(G)=\psi(g)$. In the following we will say that $U$ is convex when $\psi$ is convex. Finally, we define the global payoff function as follows: $S W: G \mapsto$ $W(G)+U(G)$, where $W$ is the total payoff function and $U$ is architecture independent and convex.

Definition 2 An efficient network $G$ is a network which maximizes the sum of the players' payoffs. In other words, network $G \in \mathcal{G}$ is efficient if $W(G) \geq W\left(G^{\prime}\right)$, for all $G^{\prime} \in \mathcal{G}$.

Definition 3 A global efficient network $G$ is a network which maximizes $S W(G)$.

We now illustrate functions that are architecture independent and convex.

Example 1.b Consumer Surplus in Cournot Oligopoly with Cost Reduction (GJ, 2003). Suppose that the inverse demand function is given by: $p\left(\sum_{i \in N} q_{i}(G)\right)=\alpha-\sum_{i \in N} q_{i}(G)$. Then the consumer surplus in $G, S C(G)$, is given by $1 / 2\left(\sum_{i \in N} q_{i}(G)\right)^{2}$. We have:

$$
1 / 2\left(\sum_{i \in N} q_{i}(G)\right)^{2}=\frac{1}{2}\left(\frac{n\left(\alpha-\gamma_{0}\right)+2 \gamma n g}{n+1}\right)^{2}
$$

For $G$ and $G^{\prime}$, such that $g=g^{\prime}$, we have $S C(G)=S C\left(G^{\prime}\right)$, so $S C$ is architecture independent. We can write $S C(G)=\psi(g)$. Moreover we have $\psi(g+1)-\psi(g)>\psi(g)-\psi(g-1)$, so $\psi$ is 
convex.

Remark 1 Let $W^{\prime}: G \mapsto W^{\prime}(G)=\sum_{i \in N} \varphi\left(g_{i}, g_{-i}\right)$. Suppose that $\phi$ and $\varphi$ satisfy Properties 1, 2 and 3. Then function $\tilde{\phi}=\phi+\varphi$ satisfies Properties 1,2 and 3 .

Example 2.b Consumer Surplus in Differentiated Cournot Oligopoly with Cost Reduction. ${ }^{11}$ Suppose that each firm $i$ faces the following linear inverse demand function: $p_{i}=\alpha-q_{i}-$ $\beta \sum_{j \in N \backslash\{i\}} q_{j}$. The consumer surplus is $\sum_{i \in N} \varphi\left(g_{i}, g_{-i}\right)$, where $\varphi\left(g_{i}, g_{-i}\right)=(1 / 2)\left[q_{i}(G)\right]^{2}$. We know that $\phi\left(g_{i}, g_{-i}\right)=\pi_{i}^{C}(G)$ satisfies Properties 1, 2 and 3. By using the same arguments as in Example 2.2 we establish that $\varphi$ satisfies Properties 1, 2 and 3. We now associate with each firm $i$ the following payoffs.

$$
\tilde{\phi}=\phi+\varphi
$$

This payoff function allows us to capture both the profit of the firm $i$ and the consumer surplus on the market $i$. Consequently, the total welfare is given by $\sum_{i \in N} \tilde{\phi}\left(g_{i}, g_{-i}\right)$. By Remark 1 ,we observe that $\tilde{\phi}$ satisfies properties 1,2 , and 3 .

\section{Results}

In this section, we establish the architectures of efficient and global efficient networks (Proposition 2). We also provide a result on the role played by the sub-modularity for the architecture of efficient networks (Proposition 3). First, we present a proposition that allows us to provide a necessary condition for networks candidate to be efficient networks. The proof of this proposition is given in the appendix.

Proposition 1 Suppose that the payoff function is given by equation 1 and $\phi$ satisfies Properties 1, 2 and 3. Moreover, suppose two subsets of players, say $\left\{i, i^{\prime}\right\}$ and $\left\{j, j^{\prime}\right\}$, with $\left\{i, i^{\prime}\right\} \neq\left\{j, j^{\prime}\right\}, g_{j} \geq g_{i}$ and $g_{j^{\prime}} \geq g_{i^{\prime}}$. If $G$ is efficient and players $i$ and $i^{\prime}$ are linked, then players $j$ and $j^{\prime}$ are linked in $G .^{12}$

\footnotetext{
${ }^{11}$ This example is based on a suggestion of Bastian Westbrock, and we would like to thank him for this.

${ }^{12}$ Note that a similar type of property has been found by GJ (Lemma 4.3, pg. 77, 2003) for networks stable against transfers. We discuss the relationship between networks stable against transfers and global efficient networks after Corollary 1.
} 
The intuition of the proof is as follows. Consider a network $G$, with $G_{i, i^{\prime}}=1$ and $G_{j, j^{\prime}}=0$. Suppose $g_{i} \leq g_{j}$ and $g_{i^{\prime}} \leq g_{j^{\prime}}$. We draw networks $G-i i^{\prime}, G$ and $G+j j^{\prime}$ in Figure 2 below, and we show that if the link $i i^{\prime}$ increases the total payoffs, then the link $j j^{\prime}$ also increases the total payoffs.

Consider players in $N \backslash\left\{i, i^{\prime}, j, j^{\prime}\right\}$. For each player $k \in N \backslash\left\{i, i^{\prime}, j, j^{\prime}\right\}$, by Property 2, the marginal payoffs she obtains when the link $j j^{\prime}$ is added to $G$ are strictly higher than the marginal payoffs she obtains when the link $i i^{\prime}$ is added to $G-i i^{\prime}$.

Consider players $i$ and $j$. First, we show that the marginal payoffs obtained by $j$ when the link $j j^{\prime}$ is added to $G, \Delta \pi_{j}^{\prime}$, are higher than the marginal payoffs obtained by $i$ when the link $i i^{\prime}$ is added to $G-i i^{\prime}, \Delta \pi_{i}$. We note that $i$ and $j$ increase their number of links by one, $j$ has formed more links in $G$ than $i$ in $G-i i^{\prime}$, and $j$ has fewer links in $G$ than $i$ in $G-i i^{\prime}$. By Properties 1 and 3 , we conclude that $\Delta \pi_{j}^{\prime} \geq \Delta \pi_{i}$. Second, we show that the marginal payoffs obtained by $i$ when the link $j j^{\prime}$ is added to $G, \Delta \pi_{i}^{\prime}$, are higher than the marginal payoffs obtained by $j$ when the link $i i^{\prime}$ is added to $G-i i^{\prime}, \Delta \pi_{j}$. We note that $i$ and $j$ deal with players who increase their number of links by one, $j$ has formed more links in $G$ than $i$ in $G-i i^{\prime}$, and $j$ has fewer links in $G$ than $i$ in $G-i i^{\prime}$. By Properties 2 and 3 , we conclude that $\Delta \pi_{i}^{\prime} \geq \Delta \pi_{j}$. It follows that the sum of the marginal payoffs of players $i$ and $j$ is higher when the link $j j^{\prime}$ is added to $G$ than when the link $i i^{\prime}$ is added to $G-i i^{\prime}$.

Consider players $i^{\prime}$ and $j^{\prime}$. Using arguments similar to those for players $i$ and $j$, we establish that the sum of the marginal payoffs of $i^{\prime}$ and $j^{\prime}$ is higher when the link $j j^{\prime}$ is added than when the link $i i^{\prime}$ is added. We conclude that if the link $i i^{\prime}$ increases the total payoffs, then the link $j j^{\prime}$ also increases the total payoffs.

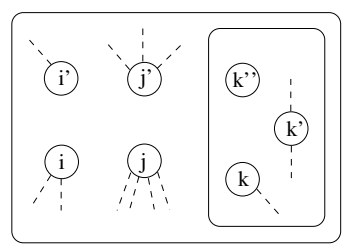

Network $G-i i^{\prime}$

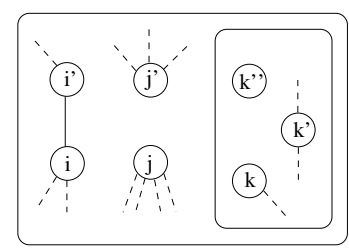

Network $G$

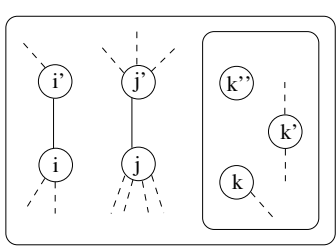

Network $G+j j^{\prime}$

Figure 2: Intuition behind Proposition 1 
Method to check whether a network is candidate for being an efficient network. We can restate Proposition 1 in a way which allows us to devise a procedure for checking whether a network is candidate for being efficient or not. Let $\bar{G}$ be the complement network of $G$, that is a network where $\bar{G}_{i, j}=1$ iff $G_{i, j}=0$. We define for each link $G_{i, j}=1$ in network $G$, the pair $m_{i, j}=\left(\min \left\{g_{i}, g_{j}\right\}, \max \left\{g_{i}, g_{j}\right\}\right)$ and for each link $\bar{G}_{i, j}=1$ in network $\bar{G}$, the pair $\bar{m}_{i, j}=\left(\max \left\{g_{i}, g_{j}\right\}, \min \left\{g_{i}, g_{j}\right\}\right)$. For each $G$, define $I(G)$ as the set of minimal pairs $m_{i, j}$ in it and $J(\bar{G})$ as the set of minimal pairs $\bar{m}_{i, j}$ in $\bar{G}$. Using Proposition 1, we observe that a network that is a candidate for being efficient has to satisfy $m_{i, i^{\prime}}+\bar{m}_{j, j^{\prime}} \not \leq(n-1, n-1)$, for all $m_{i, i^{\prime}} \in I(G)$ and $\bar{m}_{j, j^{\prime}} \in J(\bar{G})$. Let us illustrate this method through an example.

Let $N=\llbracket 1,6 \rrbracket$. First we deal with network $G$ drawn in Figure 3. We build the complement network of $G, \bar{G}$. We now determine $I(G)$ and $J(\bar{G})$, we have $I(G)=\{(2,2)\}$ and $J(\bar{G})=$ $\{(3,3),(1,5)\}$. We note that $(3,3)+(2,2)=(5,5) \leq(5,5)$, and $G$ is not a candidate for being an efficient network. Second, we consider the network $G^{\prime}$ drawn in Figure 3. We build the complement network of $G^{\prime}, \bar{G}^{\prime}$. We have $I\left(G^{\prime}\right)=\{(4,4)\}$ and $J\left(\bar{G}^{\prime}\right)=\{(1,5)\}$. We have $(4,4)+(1,5)=(5,9) \not \leq(5,5)$; hence $G^{\prime}$ is a candidate for being an efficient network.

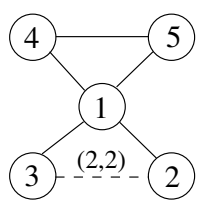

Network $G$

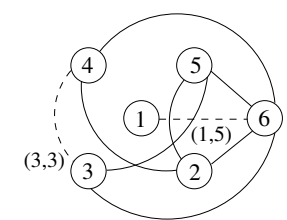

Network $\bar{G}$

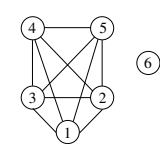

Network $G^{\prime}$

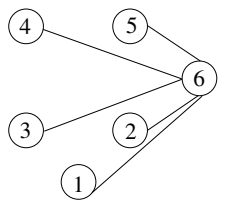

Network $\bar{G}^{\prime}$

Figure 3: Networks $G, \bar{G}, G^{\prime}$ and $\bar{G}^{\prime}$

It is worth noting that the above procedure consists in finding a minimum element from a list of pairs of degrees. It is well known that there exist polynomial time algorithms to solve such problems (see Cormen, 2001).

Corollary 1 Suppose that the payoff function is given by equation 1 and $\phi$ satisfies Properties 1, 2 and 3, and $U$ is architecture independent and convex. If $G$ is a global efficient network, and players $i$ and $i^{\prime}$ are linked in $G$, then any two players $j$ and $j^{\prime}$ whose degrees are at least as great as $i$ and $j$ are linked in $G$. 
Proof The proof is based on the same arguments as the proof of Proposition 1 and the fact that $U(G)$ is architecture independent and convex. Under the assumptions given in the proof of Proposition 1 we have $W\left(G+j j^{\prime}\right)-W(G)>W(G)-W\left(G-i i^{\prime}\right)$. Moreover, since $\psi$ is convex, we have $U\left(G+j j^{\prime}\right)-U(G) \geq U(G)-U\left(G-i i^{\prime}\right)$. We obtain $S W\left(G+j j^{\prime}\right)-S W(G)>$ $S W(G)-S W\left(G-i i^{\prime}\right) \geq 0$. The first inequality follows the addition of functions $W$ and $U$. The second inequality comes from the fact that $G$ is an efficient network. We obtain the desired contradiction.

It is worth noting that this property concerning the degrees of players has been highlighted by GJ (Lemma 4.3, pg. 77, 2003) when they examine the networks stable against transfers (firms can transfer money to their neighbors). However, global efficient networks and networks stable against transfers do not always coincide. Indeed, in networks stable against transfers the formation of an additional link $i j$ depends only on the impact of this link on the sum of the payoffs of these players. However in the context of an oligopoly, the effect of an additional link on the other firms and on the consumers is not taken into account.

Corollary 2 Suppose that the payoff function is given by equation 1 and $\phi$ satisfies Properties 1, 2 and 3, and $U$ is architecture independent and convex. Then both efficient networks and global efficient networks are interlinked stars.

Proof Let $G$ be an efficient network and let $i$ be a player who is involved in the highest number of links in $G$. To introduce a contradiction suppose that there is a player $j$ such that $g_{j} \geq 1$ and $G_{i, j}=0$. Since $i$ is involved in the highest number of links and $g_{j} \geq 1$, there exists a player $k$ such that $G_{j, k}=1$. We have simultaneously $g_{i} \geq g_{k}$ and $g_{j} \geq g_{j}$. By Proposition $1, G_{i, j}=1$, a contradiction. It follows that $G$ is an interlinked star. Since $U$ is architecture independent and convex, the result is preserved for global efficiency by using the same arguments as in the proof of Corollary 1.

Note that Corollary 2 generalizes the result of Westbrock (Proposition 1, pg. 358, 2010) to a general class of games.

In the following, we establish that efficient and global efficient networks are NSG. In other words, we establish that efficient and global efficient networks belong to a sub-class of the interlinked stars class. In Figure 4 Network $G$ is an interlinked star which is not a NSG. 

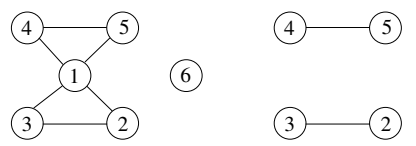

(6)

Network $G \quad$ Network $G^{-\{1\}}$

Figure 4: An interlinked star which is not a NSG

We now present a theorem which allows us to relate Proposition 1 and NSG.

Theorem 1 (Mahadev and Peled, Theorem 1.2.4, pg. 10, 1995). Suppose G such that for distinct players $i$ and $j G_{i, j}=1$ if and only if there exist non-negative parameters, $\left(w_{k}\right)_{k \in N}$ and $t$ such that $w_{i}+w_{j}>t$. Then $G$ is a NSG.

Let $D_{0}, \ldots, D_{m}$ be the degree partition of an efficient network $G$. Let us set for every $i \in D_{k}$, $w_{i}=k$ and $t=m$. By using Proposition 1, we observe that Theorem 1 is satisfied. By using Proposition 1, Corollary 1 and Theorem 1, we obtain the following proposition.

Proposition 2 Suppose that the payoff function is given by equation 1 and $\phi$ satisfies Properties 1, 2 and 3. Then every efficient network is a NSG. Similarly, if $U$ is architecture independent, every global efficient network is a NSG.

Example 1.c Cournot Oligopoly with Cost Reduction (GJ, 2003). Let us consider the individual payoff function given in Example 1.a. By Proposition 2 a network which maximizes the total profit of firms is a NSG. By Proposition 2, a network which maximizes the social welfare is a NSG.

Example 2.c Differentiated Cournot Oligopoly with Cost Reduction (Westbrock, 2010). Let us consider the individual payoff function given in Example 2.a. By Proposition 2 a network which maximizes the social welfare is a NSG.

We now relate the interlinked stars with NSG networks through an induction process by using an alternative definition of NSG.

Definition 4 Let $G$ be such that $g_{1} \geq g_{2} \geq \ldots \geq g_{n}$. Network $G$ is a NSG if $G$ is an interlinked star, and there exists $\ell \in \llbracket 1, n-1 \rrbracket$ such that $G^{-\llbracket 1, \ell^{\prime} \rrbracket}$ is an interlinked star for each 
$\ell^{\prime} \in \llbracket 1, \ell \rrbracket$ while $G^{-\llbracket 1, \ell^{\prime \prime} \rrbracket}$ is an empty network for each $\ell^{\prime \prime} \in \llbracket \ell+1, n-1 \rrbracket$.

We now establish that Definitions 1 and 4 are equivalent. Since Definition 1 clearly implies Definition 4, we show that Definition 4 implies Definition 1. In Definition 4, players in $D_{m}$ are linked with all players in $\cup_{\ell=1}^{m} D_{\ell} \cdot{ }^{13}$ Next, with Definition 4 players in $D_{m-1}$ are linked with all players in $\cup_{\ell=2}^{m-1} D_{\ell}$. Indeed, suppose that there exists $D_{k}, k \neq 1$, such that players in this set are linked with players in $D_{m}$, but not linked with players in $D_{m-1}$. Then, we obtain a contradiction since $G^{-D_{m}}$ is not an interlinked star. We can reiterate this argument for each $D_{\ell}$, with $\ell=\left\lfloor\frac{m}{2}\right\rfloor+1, \ldots, m$ for obtaining Definition 1 . In other words, to check if $G$ is a NSG we can build $G^{-\{1\}}, G^{-\llbracket 1,2 \rrbracket}, \ldots$ and check if they are interlinked stars until we find the smallest $\ell$ such that $G^{-\llbracket 1, \ell \rrbracket}$ is empty.

It is worth noting that Definition 4 implies that a NSG is an interlinked star. But the class of NSG is smaller than the class of interlinked stars provided by Westbrock. Indeed, in an interlinked star which is not a NSG, say $G$, there exist players who have not formed the number of links compatible with efficiency. The deletion of players in $G$ leads to a network $G^{\prime}$ which is not an interlinked star. Hence the player $i$, who has formed the highest number of links in $G^{\prime}$, has either formed too many links, or has not formed links with all required connected players. Network $G$ in Figure 4 is candidate for being a global efficient network according to Westbrock while it is not the case in our paper since $G$ is not a NSG: $G^{-\{1\}}$ is not an interlinked star or the empty network.

In the following, we examine the role played by Property 3. We establish that if submodularity is low relative to convexity in the first and the second argument, then efficient networks are not asymmetric. We set $\varepsilon=\max \left\{\phi\left(x^{\prime}, y\right)-\phi(x, y)-\left[\phi\left(x^{\prime}, y^{\prime}\right)-\phi\left(x, y^{\prime}\right)\right]: x^{\prime}>\right.$ $\left.x, y<y^{\prime}\right\}$. Since $\phi$ is sub-modular, $\varepsilon>0$. We now provide an assumption which allows us to restrict the set of efficient networks.

(A1): For all $(x, y) \in \llbracket 1, n-2 \rrbracket \times \llbracket 1,(n-1)(n-2) / 2 \rrbracket, \phi_{1}(x+1, y)-\phi_{1}(x, y)>2 \varepsilon$, and for all $(x, y) \in \llbracket 1, n-1 \rrbracket \times \llbracket 1,[(n-1)(n-2) / 2]-1 \rrbracket, \phi_{2}(x, y+1)-\phi_{2}(x, y)>2 \varepsilon$.

Roughly speaking the first condition in (A1) means that convexity in the first argument of $\phi$ is relatively high compared to its sub-modularity, while the second condition in (A1) states that convexity in the second argument of $\phi$ is relatively high compared to its sub-modularity.

\footnotetext{
${ }^{13}$ If the set of players who have formed $n-1$ links in non-empty, the reasoning is of the same type.
} 
Proposition 3 Suppose that the payoff function is given by equation 1 and $\phi$ satisfies Properties 3 and (A1). Then an efficient network is either empty or complete.

Proof Let $G$ be a non-empty efficient network. We show that $G$ is a complete network. To introduce a contradiction suppose it is not. Since $G$ is efficient it is a NSG which is not the complete network. There exist players $i$ and $j$ such that $G_{i} \subsetneq G_{j}$. We set $v_{j}=g_{j}-g_{i}$. Let $G^{v_{j}}$ be the network similar to network $G$ except that player $i$ is linked with each player $k$ who is linked with $j$ in $G$. Similarly, let $G^{-v_{j}}$ be the network similar to network $G$ except that player $j$ is linked only with players $k$ who are linked with $i$ in $G$. We establish that $W\left(G^{v_{j}}\right)-W(G)>$ $W(G)-W\left(G^{-v_{j}}\right)$. First, by Property 2, players $k \notin\left[\left(G_{j} \backslash G_{i}\right) \cup\{i, j\}\right]$ obtain marginal payoffs between $G^{v_{j}}$ and $G$ which are strictly higher than the marginal payoffs they obtain between $G$ and $G^{-v_{j}}$. Second, we compare the marginal payoffs associated with the changes between $G^{v_{j}}$ and $G$ for players $i$ and $j, \Delta^{+}=\phi\left(g_{i}+v_{j}, g_{-i}\right)-\phi\left(g_{i}, g_{-i}\right)+\phi\left(g_{j}, g_{-j}+v_{j}\right)-\phi\left(g_{j}, g_{-j}\right)$, and the marginal payoffs associated with the changes between $G$ and $G^{-v_{j}}$ for players $i$ and $j, \Delta^{-}=\phi\left(g_{i}, g_{-i}\right)-\phi\left(g_{i}, g_{-i}-v_{j}\right)+\phi\left(g_{j}, g_{-j}\right)-\phi\left(g_{j}-v_{j}, g_{-j}\right)$. We set $D_{i, j}=\Delta^{+}-\Delta^{-}$. Since $g_{j}=g_{i}+v_{j}$ and $g_{-i}=g_{-j}+v_{j}$, we have $D_{i, j}=\phi\left(g_{j}, g_{-i}\right)-\phi\left(g_{i}, g_{-i}\right)-\left[\phi\left(g_{j}, g_{-j}\right)-\right.$ $\left.\phi\left(g_{i}, g_{-j}\right)\right]+\phi\left(g_{j}, g_{-i}\right)-\phi\left(g_{j}, g_{-j}\right)-\left[\phi\left(g_{i}, g_{-i}\right)-\phi\left(g_{i}, g_{-j}\right)\right]$. We have $D_{i j} \geq-2 \varepsilon$. Third, we deal with the variation of marginal payoffs of players who belong to $G_{j} \backslash G_{i}$. We set $v=v_{j}-1$. The marginal payoffs obtained by each player $k \in G_{j} \backslash G_{i}$ between $G^{v_{j}}$ and $G$ are $\phi\left(g_{k}+1, g_{-k}+v\right)-\phi\left(g_{k}, g_{-k}\right)$. The marginal payoffs obtained by each player $k \in G_{j} \backslash G_{i}$ between $G$ and $G^{-v_{j}}$ are $\phi\left(g_{k}, g_{-k}\right)-\phi\left(g_{k}-1, g_{-k}-v\right)$. We set $\phi_{2}^{v}(x, y)=\phi(x, y)-\phi(x, y-v)$. We have:

$$
\begin{aligned}
\mathcal{C} & =\phi\left(g_{k}+1, g_{-k}+v\right)-\phi\left(g_{k}, g_{-k}\right)-\left[\phi\left(g_{k}, g_{-k}\right)-\phi\left(g_{k}-1, g_{-k}-v\right)\right] \\
& =\phi_{1}\left(g_{k}+1, g_{-k}+v\right)+\phi_{2}^{v}\left(g_{k}, g_{-k}+v\right)-\phi_{2}^{v}\left(g_{k}-1, g_{-k}\right)-\phi_{1}\left(g_{k}, g_{-k}\right)
\end{aligned}
$$

By (A1) $\phi_{1}\left(g_{k}+1, g_{-k}+v\right)-\phi_{1}\left(g_{k}, g_{-k}+v\right)>2 \varepsilon$ and we know that $\phi_{1}\left(g_{k}, g_{-k}\right)-\phi_{1}\left(g_{k}, g_{-k}+\right.$ $v) \leq \varepsilon$, so $\phi_{1}\left(g_{k}+1, g_{-k}+v\right)-\phi_{1}\left(g_{k}, g_{-k}+v\right)>\varepsilon+\phi_{1}\left(g_{k}, g_{-k}\right)-\phi_{1}\left(g_{k}, g_{-k}+v\right)$. It follows that $\phi_{1}\left(g_{k}+1, g_{-k}+v\right)-\phi_{1}\left(g_{k}, g_{-k}\right)>\varepsilon$. Similarly, by (A1) $\phi_{2}^{v}\left(g_{k}, g_{-k}+v\right)-\phi_{2}^{v}\left(g_{k}, g_{-k}\right)>2 \varepsilon$ and $\phi_{2}^{v}\left(g_{k}-1, g_{-k}\right)-\phi_{2}^{v}\left(g_{k}, g_{-k}\right) \leq \varepsilon$. It follows that $\phi_{2}^{v}\left(g_{k}, g_{-k}+v\right)-\phi_{2}^{v}\left(g_{k}-1, g_{-k}\right)>\varepsilon$. It follows that $\mathcal{C}>2 \varepsilon$. We conclude that $W\left(G^{v_{j}}\right)-W(G)>W(G)-W\left(G^{-v_{j}}\right)$, and $G$ is not efficient, a contradiction. 
Relationship between efficiency and stability. Under (A1), we can discuss the type of externalities that arise in the model. First, there always exist positive externalities if for all $x \in \llbracket 1, n-1 \rrbracket$ and for all $y \in \llbracket 1,[(n-2)(n-1) / 2]-1 \rrbracket$, we have $\phi_{2}(x, y)>0$. Second, by (A1) for $x \in \llbracket 1, n-1 \rrbracket$ and $y>1, \phi_{2}(x, y)-\phi_{2}(1,1)=\phi_{2}(x, y)-\phi_{2}(x, 1)+\phi_{2}(x, 1)-\phi_{2}(1,1)>2 \varepsilon-\varepsilon>0$. If for all $x \in \llbracket 1, n-1 \rrbracket$ we have $\phi_{2}(x, 1)>0$, then $\phi_{2}(x, y)>0$ for all $x \in \llbracket 1, n-1 \rrbracket$ and for all $y \in \llbracket 1,[(n-2)(n-1) / 2]-1 \rrbracket$, and there always exist positive externalities. We conclude that if no player has an unilateral incentive to remove any links in the complete network, then this network is an equilibrium (no player has an incentive to modify her strategy) and efficient network. We can use this type of arguments to establish that if for all $x \in \llbracket 1, n-1 \rrbracket$ we have $\phi_{2}(x, \llbracket 1,[(n-2)(n-1) / 2]-1)<0$, then $\phi_{2}(x, y)<0$ for all $x \in \llbracket 1, n-1 \rrbracket$ and for all $y \in \llbracket 1,[(n-2)(n-1) / 2]-1 \rrbracket$, and there always exist negative externalities. In that case, if no player has an unilateral incentive to form any links in the empty network, then this network is an equilibrium (no player has an incentive to modify her strategy) and is efficient.

Moreover, by using similar arguments as in the proof of Proposition 3, we obtain the following proposition.

Proposition 4 Suppose that the payoff function is given by equation 1 and $\phi$ satisfies Properties 1, 2 and is super-modular. If $G$ is efficient, then it is empty or complete.

Following Westbrock (pg. 365, 2010), we propose a Cournot Oligopoly model where we introduce the effect of knowledge spillovers from the whole industry on the marginal cost function. We show that if the knowledge spillovers are sufficiently high then the only networks candidates for being efficient are the complete and the empty network.

Example 3 We use the same assumptions as in Example 1, but we assume that the marginal cost function is as follows: $C\left(g_{i}\right)=\gamma_{0}-\gamma_{1} g_{i}-\gamma_{2} g_{-i}$, where $\gamma_{1}$ captures the effect of the links formed by $i$ on the marginal cost while $\gamma_{2}<\gamma_{1}$ captures the effect of knowledge spillovers on the marginal cost. Then the Cournot equilibrium output is: $q_{i}^{\star}(G)=$ $\left[\alpha-\gamma_{0}+g_{i}\left(\gamma_{1}(n-1)-\gamma_{2}(n-2)\right)+g_{-i}\left(3 \gamma_{2}-2 \gamma_{1}\right)\right] /(n+1)$. The second stage Cournot profits for firm $i \in N$ are given by $\pi_{i}^{\star}(G)=\phi\left(g_{i}, g_{-i}\right)=\left(q_{i}^{\star}(G)\right)^{2}-f\left(g_{i}\right)$. Note that $\phi$ satisfies Properties 1 and 2. Finally, if $\gamma_{2}<2 / 3 \gamma_{1}$, then $\phi$ is sub-modular, while $\phi$ is super-modular 
when $\gamma_{2} \geq 2 / 3 \gamma_{1}$. By Proposition 2, an efficient network is a NSG when $\gamma_{2}<2 / 3 \gamma_{1}$, while by Proposition 4 an efficient network is empty or complete when $\gamma_{2} \geq 2 / 3 \gamma_{1}$.

We now propose an example of a model of provision of a public good where our results can be applied.

Example 4 Provision of a public good. Consider a two-stage game example, inspired by Goyal and Joshi (2006, Example 4.1, pg. 332). In the first stage of this game, players form links. In the second stage, each player chooses a level of costly effort to produce a good. The effort of player $i$ allows her to increase her payoffs, and generates positive externalities or spillovers for the payoffs of other players.

In the first stage, we assume that each player owns an amount of time equal to $m>n-1$. She chooses to allocate this time between two activities. The first activity consists in forming or maintaining links with other players. The links formed by player $i$ allow her to decrease the cost of her own effort. We assume that each link takes one unit of time to be formed or maintained. The second activity takes into account the fact that spillovers are not a free good. ${ }^{14}$

It follows that the payoff function of each player $i$ depends on her own effort, the effort of the other players and the links that she has formed in the network. Given the network formed at the first stage, the payoff function of each player at the second stage is:

$$
S_{i}\left(e_{i}, e_{-i}\right)={\frac{\left(e_{i}\right)^{2}}{2}}^{2} \frac{\left(m-g_{i}\right)}{8} \sum_{j \neq i}\left(e_{j}-1\right)+\gamma\left(\sum_{j \neq i}\left(e_{j}-1\right)\right)^{2}-\frac{\left(e_{i}\right)^{3}}{3\left(g_{i}+1\right)}
$$

The term $\frac{\left(m-g_{i}\right)}{8}\left(\sum_{j \neq i} e_{j}-1\right)$ captures the spillovers obtained by player $i$ from the others due to the amount of time she spends on absorbing spillovers. The term $\gamma\left(\sum_{j \neq i}\left(e_{j}-1\right)\right)^{2}$ captures the spillovers obtained by player $i$ which does not depend on the choice of $i$, with $\gamma>0$. Finally, the term $\left(e_{i}\right)^{3} / 3\left(g_{i}+1\right)$ captures the positive impact of the formation of links on the cost of player $i$ 's effort.

\footnotetext{
${ }^{14}$ This activity consists in increasing what Cohen and Levinthal (1990) called the absorptive capacity, that is the capacity to capture spillovers "in the air".
} 
The equilibrium effort at the second stage is $e_{i}=g_{i}+1$. Since $\sum_{j \neq i} g_{j}=2 g_{-i}+g_{i}$, we can write the payoff function of each player $i$ at the first stage as:

$$
\pi_{i}\left(g_{i}, g_{-i}\right)=\frac{\left(g_{i}+1\right)^{2}}{2}+\frac{\left(m-g_{i}\right)}{8}\left(2 g_{-i}+g_{i}\right)+\gamma\left(2 g_{-i}+g_{i}\right)^{2}-\frac{\left(g_{i}+1\right)^{2}}{3} .
$$

Since $\gamma>0, \pi_{i}\left(g_{i}, g_{-i}\right)$ is convex in its first and in its second argument. Moreover, if $\gamma \leq 1 / 16$, then $\pi_{i}\left(g_{i}, g_{-i}\right)$ is sub-modular while it is super-modular if $\gamma \geq 1 / 16$. By Proposition 2, an efficient network is a NSG when $\gamma<1 / 16$, while by Proposition 4 an efficient network is empty or complete when $\gamma \geq 1 / 16$.

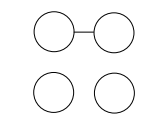

Network $G^{1}$

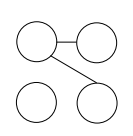

Network $G^{2}$

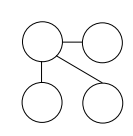

Network $G^{3}$

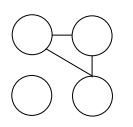

Network $G^{4} \quad$ Network $G^{5}$

Figure 5: Some specific networks

\section{Discussion}

We now examine the role played by Property 1 for efficient networks. To simplify we deal with the following specific profit function gross of costs of forming links: $\Pi_{i}(G)=a\left(g_{i}\right)^{2}-b g_{i} g_{-i}+$ $c\left(g_{-i}\right)^{2}$, with $a>0, b=2$ and $c=1$, and the following function of costs of forming links: $f\left(g_{i}\right)=\tau\left(g_{i}\right)^{2}, 0 \leq \tau \leq a$. Thus, the payoff function of each player $i$ is $\pi_{i}(G)=\phi\left(g_{i}, g_{-i}\right)=$ $(a-\tau)\left(g_{i}\right)^{2}-2 g_{i} g_{-i}+g_{-i}^{2}$. It is worth noting that in this situation, the parameter $\tau$ decreases the convexity in the first argument of $\phi$. We set $N=\llbracket 1,4 \rrbracket$. If $a=\tau$, then networks $G^{1}$ and $G^{2}$ shown in Figure 5 are efficient; if $a-\tau=0.25$, then network $G^{2}$ shown in Figure 5 is efficient; if $a-\tau=0.5$, then networks $G^{3}$ and $G^{4}$ shown in Figure 5 are efficient; if $a-\tau>1.5$, then network $G^{5}$ shown in Figure 5 is efficient. To sum up, in the example described here, when convexity in the first argument of $\phi$ increases (here it is equivalent to decreasing the costs of forming links), the number of links increases in efficient networks.

We can now observe that the convexity in the first argument of $\phi(\cdot, y)$ is in general very different from the convexity of $\phi\left(\cdot, y^{\prime}\right), y \neq y^{\prime}$. This difference in convexity explains the emergence of some specific architectures. Suppose for instance that $\phi(\cdot, 0)$ is very convex relative to $\phi(\cdot, y)$, 
$y \neq 0$, and convexity in the second argument and sub-modularity are sufficiently low. Then, star network (network $G^{3}$ in Figure 5), which is particularly asymmetric, is the unique efficient network. We illustrate this point through the following example. We set $N=\llbracket 1,4 \rrbracket$ and we let the payoff function $\phi\left(g_{i}, g_{-i}\right)$ be sum up through the following matrix:

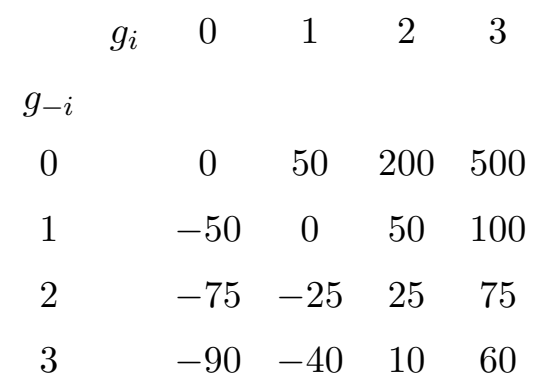

Suppose we have $\phi(1,0)=50$. In such a situation network $G^{3}$ in Figure 5, which is the star network, is efficient.

\section{Appendix}

Proof of Proposition 1. Let $G$ be an efficient network. To introduce a contradiction, suppose that there exist two subsets of players, say $\left\{i, i^{\prime}\right\}$ and $\left\{j, j^{\prime}\right\}$ with $\left\{i, i^{\prime}\right\} \neq\left\{j, j^{\prime}\right\}$, in $G$ such that $G_{i, i^{\prime}}=1, G_{j, j^{\prime}}=0, g_{i} \leq g_{j}$ and $g_{i^{\prime}} \leq g_{j^{\prime}}$. It is worth keeping in mind that if $k \in N$ is involved in $g_{k}$ links, then there are $g_{-k}=g-g_{k}$ links in which $k$ is not involved. It follows that if $g_{i} \leq g_{j}$ and $g_{i^{\prime}} \leq g_{j^{\prime}}$, then $g_{-i} \geq g_{-j}$ and $g_{-i^{\prime}} \geq g_{-j^{\prime}}$ in $G$. Since $\left\{i, i^{\prime}\right\} \neq\left\{j, j^{\prime}\right\}$, we have two possibilities, (i) either players $i, j, i^{\prime}, j^{\prime}$ are 4 distinct players, (ii) or there are 3 distinct players. (i) First, we deal with the case where players $i, j, i^{\prime}, j^{\prime}$ are distinct players: $\left\{i, i^{\prime}\right\} \cap\left\{j, j^{\prime}\right\}=\emptyset$. In the following, we denote by $N^{\prime}=N \backslash\left\{i, j, i^{\prime}, j^{\prime}\right\}$. Let us compare $W\left(G+j j^{\prime}\right)$ and $W(G)$ :

$$
\begin{aligned}
\Gamma^{+}=W\left(G+j j^{\prime}\right)-W(G)= & \phi_{1}\left(g_{j}+1, g_{-j}\right)+\phi_{1}\left(g_{j^{\prime}}+1, g_{-j^{\prime}}\right)+\phi_{2}\left(g_{i^{\prime}}, g_{-i^{\prime}}+1\right) \\
& +\phi_{2}\left(g_{i}, g_{-i}+1\right)+\sum_{\ell \in N^{\prime}} \phi_{2}\left(g_{\ell}, g_{-\ell}+1\right) .
\end{aligned}
$$

We now compare $W(G)$ and $W\left(G-i i^{\prime}\right)$ : 


$$
\begin{aligned}
\Gamma^{-}=W(G)-W\left(G-i i^{\prime}\right)= & \phi_{1}\left(g_{i}, g_{-i}\right)+\phi_{1}\left(g_{i^{\prime}}, g_{-i^{\prime}}\right)+\phi_{2}\left(g_{j}, g_{-j}\right) \\
& +\phi_{2}\left(g_{j^{\prime}}, g_{-j^{\prime}}\right)+\sum_{\ell \in N^{\prime}} \phi_{2}\left(g_{\ell}, g_{-\ell}\right) .
\end{aligned}
$$

Since $G$ is efficient, we have $W(G)-W\left(G-i i^{\prime}\right) \geq 0$ and $W\left(G+j j^{\prime}\right)-W(G) \leq 0$. To obtain a contradiction it is sufficient to show that $W\left(G+j j^{\prime}\right)-W(G)>W(G)-W\left(G-i i^{\prime}\right)$. First, we deal with $\mathcal{A}_{1}(G)=\sum_{\ell \in N^{\prime}} \phi_{2}\left(g_{\ell}, g_{-\ell}+1\right)-\sum_{\ell \in N^{\prime}} \phi_{2}\left(g_{\ell}, g_{-\ell}\right)$. We have $\mathcal{A}_{1}(G)>0$ since $\phi$ is strictly convex in its second argument. We now deal with $\mathcal{A}_{2}(G)=\phi_{1}\left(g_{j}+1, g_{-j}\right)-\phi_{1}\left(g_{i}, g_{-i}\right)$. We have $\mathcal{A}_{2}(G) \geq 0$ since $\phi_{1}\left(g_{j}+1, g_{-j}\right) \geq \phi_{1}\left(g_{j}+1, g_{-i}\right) \geq \phi_{1}\left(g_{i}+1, g_{-i}\right) \geq \phi_{1}\left(g_{i}, g_{-i}\right)$. The first inequality comes from the sub-modularity of $\phi$. The second and the third inequalities come from the convexity of $\phi$ in its first argument. We use the same arguments to show that $\mathcal{A}_{3}(G)=$ $\phi_{1}\left(g_{j^{\prime}}+1, g_{-j^{\prime}}\right)-\phi_{1}\left(g_{i^{\prime}}, g_{-i^{\prime}}\right) \geq 0$. We now deal with $\mathcal{A}_{4}(G)=\phi_{2}\left(g_{i}, g_{-i}+1\right)-\phi_{2}\left(g_{j}, g_{-j}\right)$. We have $\mathcal{A}_{4}(G)>0$ since $\phi_{2}\left(g_{i}, g_{-i}+1\right)>\phi_{2}\left(g_{i}, g_{-j}\right) \geq \phi_{2}\left(g_{j}, g_{-j}\right)$. The first inequality comes from the convexity of $\phi$ in its second argument. The second inequality comes from the sub-modularity of $\phi$. We use the same arguments to show that $\mathcal{A}_{5}(G)=\phi_{2}\left(g_{i^{\prime}}, g_{-i^{\prime}}+\right.$ 1) $-\phi_{2}\left(g_{j^{\prime}}, g_{-j^{\prime}}\right)>0$. To sum up, we have: $W\left(G+j j^{\prime}\right)-W(G)-\left[W(G)-W\left(G-i i^{\prime}\right)\right]=$ $\sum_{r=1}^{5} A_{r}(G)>0$, since $A_{r}(G) \geq 0$, for $r \in \llbracket 1,5 \rrbracket$, and $A_{1}(G), A_{4}(G), A_{5}(G)>0$. It follows that $W\left(G+j j^{\prime}\right)-W(G)>W(G)-W\left(G-i i^{\prime}\right) \geq 0$ and $G$ is not an efficient network, a contradiction.

(ii) Second, we deal with the case where there are 3 distinct players, that is $\left\{i, i^{\prime}\right\} \neq\left\{j, j^{\prime}\right\}$ and $\left\{i, i^{\prime}\right\} \cap\left\{j, j^{\prime}\right\} \neq \emptyset$. We assume that $i=j$. We have $G_{i, i^{\prime}}=1, G_{i, j^{\prime}}=0$, and $g_{i^{\prime}} \leq g_{j^{\prime}}$. We note that $W\left(G+i j^{\prime}\right)-W(G)$ is similar to $\Gamma^{+}$except that $\phi_{1}\left(g_{j}+1, g_{-j}\right)=\phi_{1}\left(g_{i}+1, g_{-i}\right)$, and $\phi_{2}\left(g_{i}, g_{-i}+1\right)=0$. Moreover, $W(G)-W\left(G-i i^{\prime}\right)$ is similar to $\Gamma^{-}$except that $\phi_{2}\left(g_{j}, g_{-j}\right)=0$. By using the same argument as in the four distinct players case, we conclude. Similar proofs can be done when we permute the identity of the players. 


\section{References}

[1] M. Belhaj, S. Bervoets, and F. Deroian. Network design under local complementarities. Working paper, 2013.

[2] P. Billand, C. Bravard, S. Chakrabarti, and Sarangi S. A note on networks of collaboration in multi-market oligopolies. The Annals of Regional Science, 53:325-335, 2014.

[3] A. Calvó-Armengol and M. O. Jackson. The effects of social networks on employment and inequality. American Economic Review, 94(3):426-454, June 2004.

[4] W.M. Cohen and D.A. Levinthal. Absorptive capacity: A new perspective on learning and innovation. Administrative Science Quaterly, 35:128-152, 1990.

[5] T. H. Cormen, C. Stein, R. L. Rivest, and C. E. Leiserson. Introduction to Algorithms. McGraw-Hill Higher Education, 2nd edition, 2001.

[6] S. Goyal and S. Joshi. Networks of collaboration in oligopoly. Games and Economic Behavior, 43(1):57-85, 2003.

[7] S. Goyal and S. Joshi. Unequal connections. International Journal of Game Theory, 34(3):319-349, 2006.

[8] M. Granovetter. Getting a job. Harvard University Press, 1974.

[9] J. Hagedoorn. Inter-firm R\&D partnerships: An overview of major trends and patterns since 1960. Research Policy, 31(4):477-492, 2002.

[10] M. König, S. Battiston, M. Napoletano, and F. Schweitzer. The efficiency and stability of R\&D networks. Games and Economic Behavior, 75:694-713, 2012.

[11] M. König, C. Tessone, and Y. Zénou. A dynamic model of network formation with strategic interactions. Working Paper, 2011.

[12] N. Mahadev and U. Peled. Threshold Graphs and Relative Topics. Elsevier, 1995.

[13] W.W. Powell, D.R. White, K.W. Koput, and J. Owen-Smith. Network dynamics and field evolution: The growth of interorganizational collaboration in the life sciences. American Journal of Economics and Sociology, 110(4):1132-1205, 2005.

[14] B. Westbrock. Natural concentration in industrial research collaboration. The RAND Journal of Economics, 41(2):351-371, 2010. 